\title{
10.5005/jp-joumals-10009-1285 \\ CASE REPORT \\ The Assessment of Fetal Behavior of a Fetus with Lissencephaly by 4D Ultrasound
}

\author{
Alin Başgül Yiğiter, Gökçenur Gönenç, Herman İşçi, Nilgün Güdücü
}

\begin{abstract}
Lissencephaly is malformation of gyral and sulcal structures of the cerebrum as a result of abnormal neuronal migration. The most common clinical manifestations of lissencephaly are severe psychomotor retardation, developmental defects, seizures, failure to thrive, and infant or early childhood deaths. Because direct assessment of functional development of the central nervous system in fetus and infant is not feasible, patients with lissencephaly who survive infancy are usually not diagnosed until childhood. Recently, the analysis of fetal behavior has been proposed as a measure of neurological maturation, which would be valuable in the diagnostic pathway of lissencephaly. In our knowledge the assessment of fetal behavior in a fetus with lissencephaly was not published yet in the literature.
\end{abstract}

Keywords: Fetal behavior, Lissencephaly, 4D ultrasound, Fetal movements.

How to cite this article: Y iğiter $A B, G$ önenç $G$, İşçi $H, G$ üdücü $\mathrm{N}$. The Assessment of Fetal Behavior of a Fetus with Lissencephaly by 4D Ultrasound. Donald School J Ultrasound Obstet G ynecol 2013;7(2):208-212.

\section{Source of support $\mathrm{Nil}$}

\section{Conflict of interest: None declared}

\section{INTRODUCTION}

The development of cerebral cortex occurs in three consecutive steps: Cell proliferation, neuronal migration and organization. ${ }^{1} \mathrm{~A}$ defect in the process of neuronal migration between eighth and 14th gestational weeks may result in gyral and sulcal malformation, which is referred to as lissencephaly. ${ }^{2}$

The most common clinical manifestations of lissencephaly are severe psychomotor retardation, developmental defects, seizures, failure to thrive and infant or early childhood deaths. ${ }^{3}$ B ecause direct assessment of functional development of the central nervous system (CNS) in fetus and infant is not feasible, patients with lissencephaly who survive infancy are usually not diagnosed until childhood. ${ }^{4}$ Recently, the analysis of fetal behavior has been proposed as a measure of neurological maturation, ${ }^{5}$ which would be valuable in the diagnostic pathway of lissencephaly. Likewise, increasing data in the field of fetal and neonatal neurology point to the very possible role of a new prenatal screening test for the assessment of fetal behavior, K urjak's antenatal neurobehavioral test (KANET) in diagnosis of various CNS disorders. ${ }^{6}$
The anatomic definition of intracranial structures by radiological studies is challenging. Therefore, the use of fetal behavioral patterns was proposed to fill this diagnostic gap. ${ }^{7}$ In fact, the introduction of three- and four-dimensional (4D) ultrasonography have been the turning point in the assessment of fetal behavior. ${ }^{8}$ It is now feasible to comprehend the morphological dynamics, such as yawning, smiling, blinking, that may be used to assess neurophysiological development, as well as to detect pathological anatomy, with these sophisticated radiological tools. ${ }^{9}$ Besides, fetal facial expressions and the quality of fetal movements can be assessed by $4 \mathrm{D}$ ultrasound, which may be evaluated along with the quantity of fetal movements that are detected by two-dimensional (2D) ultrasound. ${ }^{10}$ Understanding the relationship between fetal behavior and brain developmental processes in different periods of gestation allows to distinguish between normal and abnormal CNS development. ${ }^{11}$

Pregnancies complicated with neurologically abnormal fetuses, intrauterine growth restricted fetuses, and diabetes mellitus have been evaluated by $4 D$ sonography to detect fetal behavioral states in the last decade. ${ }^{12}$ The results of the studies reveal that complicated fetuses demonstrate different behavioral types. That is why we applied KANET to a fetus with lissencephaly. In the following KANET is summarized: It is a new scoring system for fetal neurobevaior which is based on prenatal assessment of the fetus by 3D/4D ultrasonography. ${ }^{13}$ In 2010, the test was standardized in order to be applied easily by fetal medicine specialists. A ccording to this standardization KANET should be performed between 28 and 38th week of gestation. The duration of the examination should be between 15 and 20 minutes concurently with an awake fetus. ${ }^{14}$

In this case report, a fetus with lissencephaly diagnosed antenatally by means of abnormal fetal behavior assessed by using $4 \mathrm{D}$ ultrasonography is presented.

\section{CASE REPORT}

A 26-year-old gravida 1, para 0 asymptomatic woman was seen for routine pregnancy follow-up at the 20th gestational week. Her medical history was unremarkable. She had been previously seen for routine pregnancy follow-up examinations at the 6th, 10th, 12th and 16th gestational weeks, all 
of which were found to be normal. Sonographic scan performed at the 20th gestational week revealed mild ventriculomegaly with $11 \mathrm{~mm}$, severe polyhydramnios and symetric intrauterine growth restriction. Upon these findings, the necessity of karyotype analysis was discussed with the patient. A fter an informed consent had been obtained, amniosynthesis was carried out. K aryotype analysis was normal.

Sonographic examination performed at the 30th gestational week showed double layered cerebral cortex, incomplete insula opercularization (the failure of operculum formation which is a part of cerebrum bordering to cover the sylvian fissure and concealing the insula), and the abscence of gyral formation in the cerebral cortex (Figs 1 and 2). Fetal behavior was assessed using 4D sonography and revealed invariable hand movements (Fig. 3). The hands
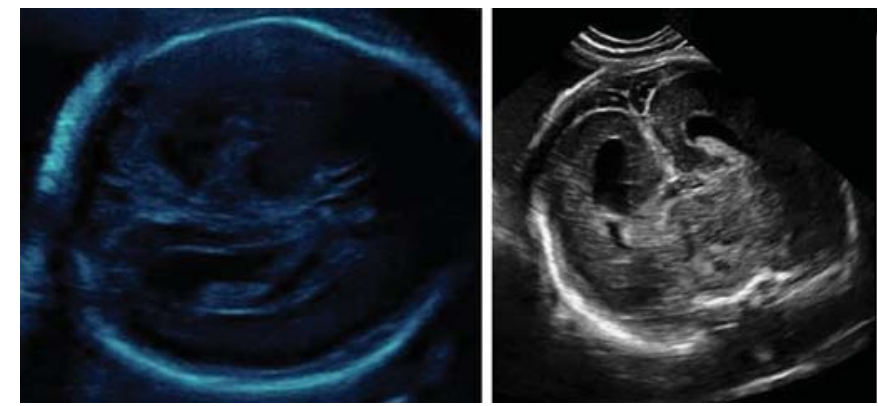

Fig. 1: 2D ultrasonographic image of a fetus with lissencephaly showing fetal cranium on transverse section (on the right) and coronal section (on the left) at 31st weeks gestation. Mild ventriculomegaly was noted and smooth brain with no gyral formation is visualized

of the fetus were cramped with clenched fists (Fig. 4). Though seldom and slow mounting was detected (Fig. 5), the stomach could not be visualized likely due to impaired
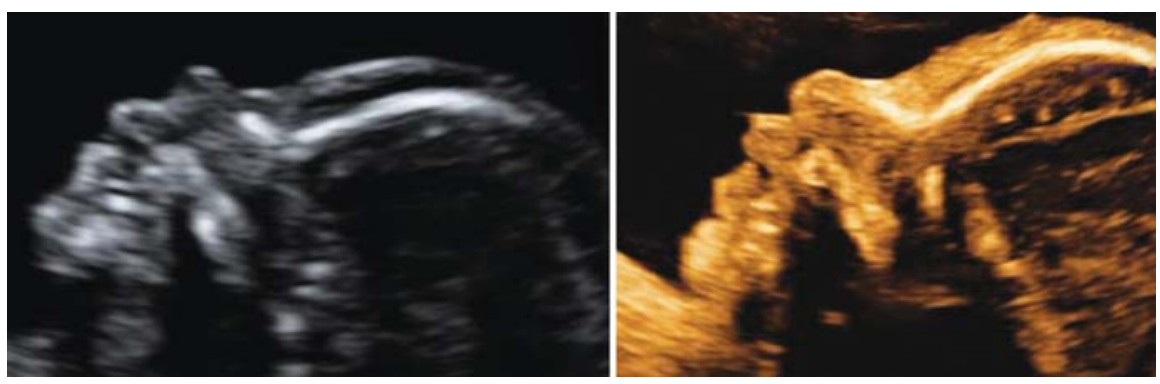

Fig. 2: $2 \mathrm{D}$ ultrasonography of a fetus with lissencephaly at 21st week (on the right) and 31st week (on the left) gestation
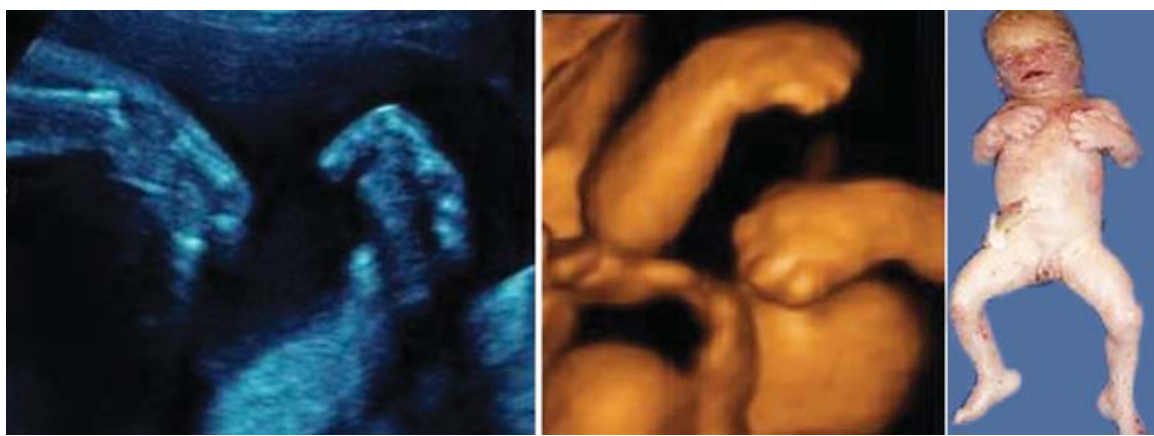

Fig. 3: 2D ultrasonographic (on the right) and 3D ultrasonographic (in the middle) view of bilateral clenched hands and postmortem same behavior pattern of the fetus with lissencephaly (on the left)

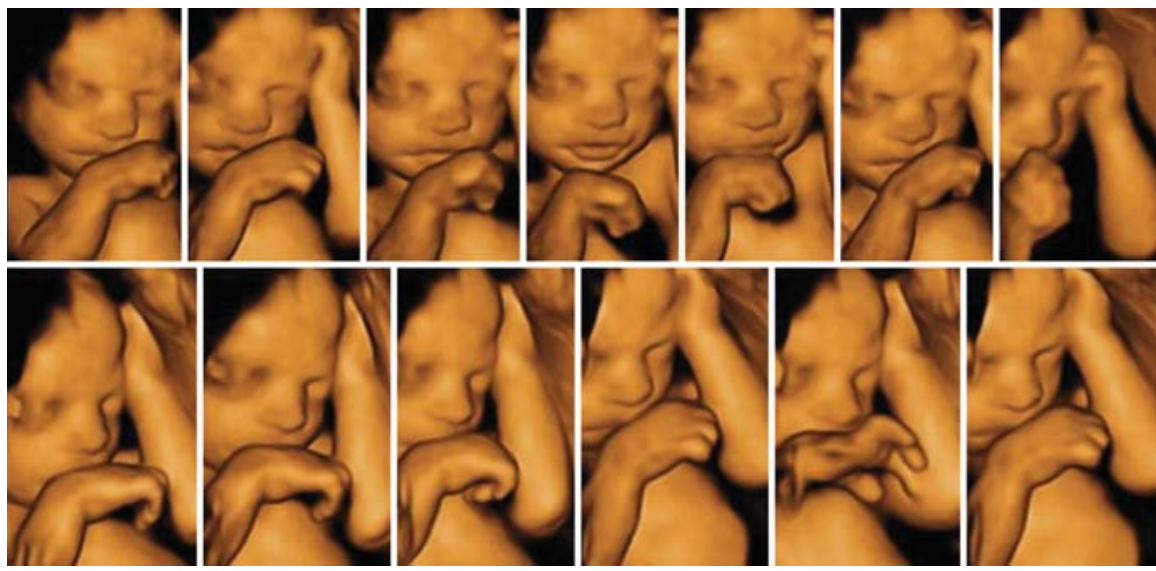

Fig. 4: 4D serial images of the fetus with lissencephaly. Bilateral clenched hands with rare and limited opening is seen 


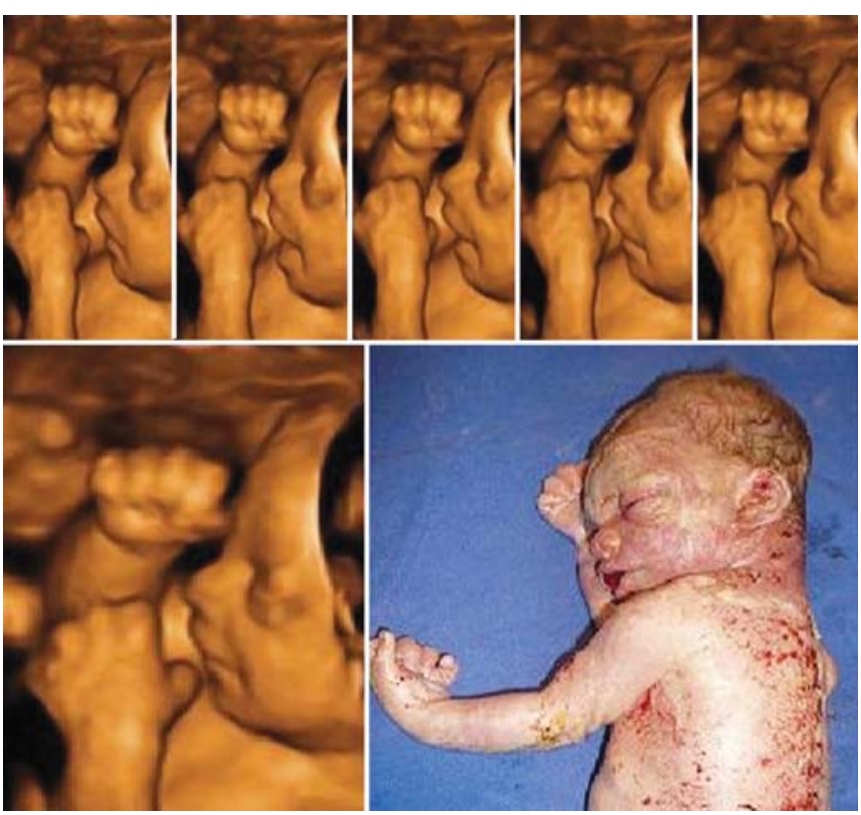

Fig. 5: 4D serial ultrasonographic images of a fetus with lissencephaly; seldom opening of the mouth, eye movements and bilateral clenched hands (upper and lower right) and postmortem same behavioral pattern with limited opened mouth, eye and clenched hands (lower left) are seen

swallowing (Fig. 6). A nteflexion of the head was seldom. Sutures and head circumference were normal. Isolated eye blinking and facial alterations were extremely rare. Hand movements and leg movements had poor repertoire. Leg movements were jerky and the flexion of the legs were not visualized during examination (Fig. 7). A mniodrainage was performed at the 31stgestational week. M agnetic resonance imaging of the fetal cranium revealed lissencephaly. The parents were fully informed about the prognosis of lissencephaly and therapeutic options. Because the parents chose termination of the pregnancy, the fetus was terminated. Autopsy confirmed the diagnosis of lissencephaly.

\section{DISCUSSION}

During fetal growth all systems follow the growing process. The progression of CNS development of the fetus is widening the repertoire of fetal movements by the time.

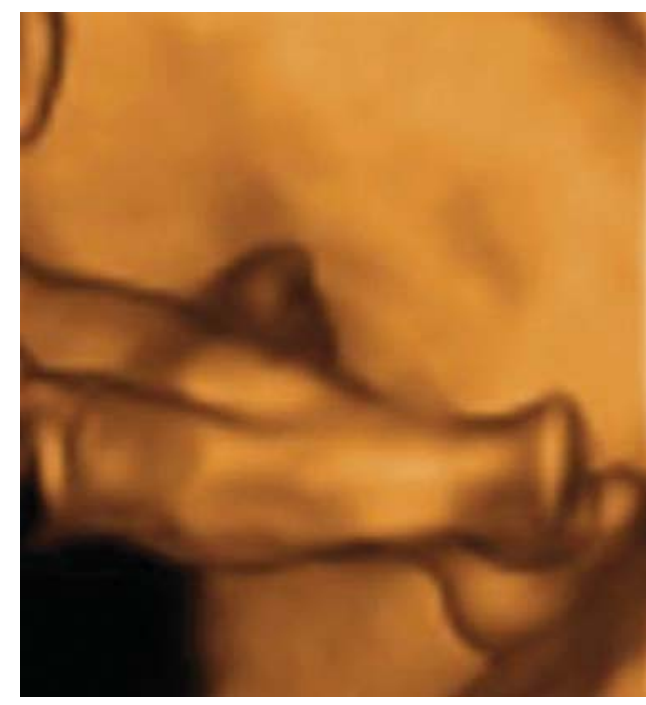

Fig. 7: 3D ultrasonographic appearance of the legs of a fetus with lissencephaly. Note: The straight appearance of the legs

The establishment of neural connections is pronounced by new behavioral patterns or enrichment of the existing. As a result, the defragmentation of random and profuse fetal activity into organized form, corralates with the maturation of CNS. ${ }^{15}$

Previously fetal behavior of an anencephalic fetus compared with a normal one was published in the literature. ${ }^{16}$ In the normal fetus, the movement patterns of the hand were continuous and variable in direction. Hand movement around the mouth and other movements of the hand to specific body parts can be observed in a normal fetus, but these characteristic patterns did not appear in the anencephalic fetus. M ovements of the hand occured only in one direction (hand to head) and it was abnormal, forceful and jerky. There were almost absent movements of the lower extremities. All the frequencies of the movement patterns in the anencephalic fetus w ere decreased compared with normal longitudinal parameter in all trimesters. Similar to anencephalic fetus, the lissencephalic fetus had a poor repertoire of hand movements which occured in one direction (hand to head) with a jerky pattern, and decreased lower extremity movements are also similar to an anencephalic fetus likely due to abnormal neurological status.
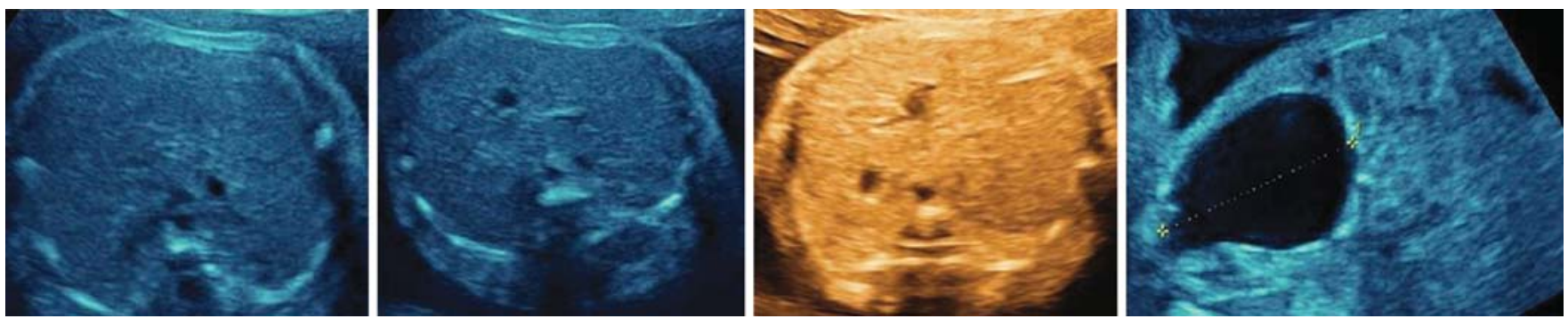

Fig. 6: 2D ultrasonographic images showing absence of the stomach at different gestational weeks and visible bladder is noted on the left 
The Assessment of Fetal Behavior of a Fetus with Lissencephaly by 4D Ultrasound

A ccording to KA NET scoring system before the Osaka consensus meeting in 2011, a prenatal score between 14 and 20 reveals normal neonates, a prenatal score between 5 and 13 reveals mildly or moderately abnormal neonates, whereas a prenatal score below 5 reveals neurologically abnormal infants. ${ }^{13} \mathrm{~N}$ ow the revised K A NET scoring system is used, with scores abnormal 0-5, borderline 6-9 and normal 10-16 points. The case we presented was scored according to the KANET after revision with eight parameters, and had abnormal KANET score which was 5. Anteflexion of the head was in small ranges (one point), head circumference was normal (one point), eye blinking was not noted, facial alterations were not present but mouth opening was noted without fluency (one point), isolated hand movements were in poor repertoire with abrupt hand to face movements (one point), isolated leg movements had also poor repertoire (one point), fingers were clenched bilateral and gestalt perception of general movements were abnormal. The most important clue in sonographic scan in the present case was clenched hands with rare and limited opening, isolated decreased hand and leg movements with a jerky patern and lack of complex movements. Parallel to our case, Kurjak et al reported poor repertoire of hand and finger movements in three fetuses with arthrogryposis congenita, vermis aplasia and a fetus with previous sibling with verified cerebral palsy. ${ }^{6}$

A ndonotopo and $\mathrm{K}$ urjak compared fetal behavior of 50 normal and 50 growth restricted fetuses. ${ }^{17}$ Poor repertoire of movements was detected in the growth restricted group whereas movements of the normal fetuses were complex and variable in speed and composition. Head and hand movements were monotone and were performed slowly with small amplitude in the growth restricted group. Position changes were more often in normal fetuses. The future use of $4 D$ sonography to assess fetal behavior as a possible indicator of fetal neurological condition in growth restricted fetuses is encouraged.

A hmed et al used 4D sonography to establish fetal behavioral profile in high-risk pregnancies. ${ }^{18}$ They detected excessive hypertonic movements in the fetuses with anencephaly and encephalocele whereas fetuses affected by homocystinuria and Meckel-Gruber syndrome demonstrated hypotonia.

A delay of the appearance of fetal movement patterns by 1 to 2 weeks has been found in generally well-controlled studies in women with type 1 diabetes mellitus. ${ }^{19} \mathrm{~A}$ bnormal movement paterns were detected in fetuses with abnormalities (anencephaly, encephalocele, intrauterine growth restriction). ${ }^{16-18}$ Reported abnormal movement patterns are indicative of altered brain and muscular development and are most commonly due to changes in the quality of the movements (e.g. abrupt, forceful movements or slow, monotonous movements). In some fetuses this coincides with a change in the quantity of the movements, mostly a decrease. Unfortunately, it is still not feasible to use quality of movements in a single case as an instrument for clinical decision-making. ${ }^{20}$

Although the assessment of the fetal behavior with 4D ultrasound is of paramount importance when monitorization of fetal neurodevelopment is necessary prenatally, it is considerably time-consuming. ${ }^{18}$ On the other hand, the learning curve is relatively short for the obstetricians who have al ready been expertized in obstetrical ultrasound, and therefore the duration of the test can be reduced to a reasonable degree, ranking from 15 to 20 minutes. ${ }^{21}$ This time factor appears rather insignificant in relation to the importance of prenatal diagnosis of CNS disorders with their specific medicolegal implications.

\section{CONCLUSION}

Understanding the relationship between fetal behavior and the developmental processes of the CNS can allow to distinguish between normal and abnormal CNS devel opment. Early diagnosis of various structural and also functional abnormalities in utero will be available. Whereupon we will be able to predict antenatally which fetuses are at risk for adverse neurological outcomes irrespective of intrapartum management. We conclude that a fetus with lissencephaly demonstrates abnormal fetal behavior especially with the clenched hand and a decrease in both facial alterations and complex movements.

\section{REFERENCES}

1. Leventer RJ, Guerrini R, D obyns W B. M alformations of cortical development and epilepsy. Dialogues Clin Neurosci 2008;10(1):47-62.

2. K undaragi NG, Taori $K$, Disawal A, Jathar $C, V$ aghasiya $D$. L issencephaly with bilateral complete cleft lip and palate: An early second-trimester diagnosis. J U Itrasound M ed 2012 Jan;31(1):123-29.

3. A shwal S. Congenital structural defects. In: Swaiman KF, A shwal S (Eds). Pediatric neurology: Principles and practice. St. Louis: Mosby; 1999.

4. Dobyns W B, Das S.In: Pagon RA, BirdTD, D olan CR, Stephens K, A dam M P. LIS1-associated lissencephaly/subcortical band heterotopia. Seattle (W A): U niversity of W ashington, Seattle; 1993.

5. Prechtl HF. Qualitative changes of spontaneous movements in fetus and preterm infant are a marker of neurological dysfunction. Early Hum Dev 1990 Sep;23(3):151-58.

6. Kurjak A, A bo-Y aqoub S, Stanojevic M, Y igiter A B, V asilj 0 , $L$ ebit $D$, et al. The potential of $4 D$ sonography in the assessment of fetal neurobehavior-multicentric study in high-risk pregnancies. J Perinat M ed 2010;38(1):77-82. 
7. Nijhuis JG (Ed). Fetal behaviour: Developmental and perinatal aspects. Oxford: Oxford U niversity Press, 1992:283.

8. Salihagic-K adic A, Kurjak A, M ediæ M, A ndonotopo W, A zumendi G. New data about embryonic and fetal neurodevelopment and behavior obtained by 3D and 4D sonography. J Perinat M ed 2005;33(6):478-90.

9. Kurjak A, Vecek N, Hafner T, B ozek T, Funduk-Kurjak B, Ujevic B. Prenatal diagnosis: What does four-dimensional ultrasound add? J Perinat M ed 2002;30(1):57-62.

10. Y igiter $A B, K$ avak ZN. Normal standards of fetal behavior assessed by four-dimensional sonography. J M atern Fetal Neonatal Med 2006 N ov; 19(11):707-21.

11. Lebit DF, Vladareanu PD. The role of $4 D$ ultrasound in the assessment of fetal behaviour. Maedica (Buchar) 2011 A pr;6(2):120-27.

12. Kurjak A. Tikvica A, Miskovic B, Stanojevic M, A hmed B, $V$ asilj 0 . Fetal behavior in high-risk pregnancies assessed by two-dimensional (2D) and three-dimensional/four-dimensional (3D)/(4D) Ultrasound: A review. DSJUOG 2008 Jul-Sep; 2(3):35-47.

13. Kurjak A, M iskovic B, Stanojevic M, A miel-Tison C, A hmed $B$, Azumendi $G$, et al. New scoring system for fetal neurobehavior assessed by three- and four-dimensional sonography. J Perinat M ed 2008;36(1):73-81.

14. Stanojevic M, Talic A, M iskovic B, Vasilj O, Shaddad A N, Predojevic M , et al. A n A ttepmt to standardize K urjak's antenatal neurodevelopmental test: O saka Consensus Statement. DSJ U OG 2011 Oct-Dec;5:317-29.

15. Salihagic-Kadic A, Medic M, Kurjak A, Andonotopo W, A zumendi $G, H$ afner $T$, et al. Four-dimensional sonography in the assessment of fetal functional neurodevelopment and behavioral patterns. UItrasound Rev Obstet Gynecol 2005;5(2):1-15.

16. A ndonotopo W, Kurjak A, Kosuta MI. Behavior of an anencephalic fetus studied by $4 \mathrm{D}$ sonography. J M atern Fetal Neonatal Med 2005 Feb;17(2):165-68.

17. A ndonotopo W, K urjak A. The assessment of fetal behavior of growth restricted fetuses by $4 \mathrm{D}$ sonography. J Perinat $\mathrm{Med}$ 2006;34(6):471-78.
18. Badreldeen A, K urjak A, A ndonotopo W, K henyab N, Saleh N, A I-M ansori Z. Fetal behavioral and structural abnormalities in high risk fetuses assessed by $4 D$ sonography U Itrasound Rev Obstet Gynecol 2005 Dec;5(4):275-87.

19. M ulder EJ, V isser GH. G rowth and motor development in fetuses of women with type-1 diabetes. II. Emergence of specific movement patterns. Early Hum Dev 1991 M ay;25(2):107-15.

20. Visser GH. A bnormal fetal movements. Ultrasound Obstet Gynecol 1992;2:3-5.

21. M iskovic B, V asilj 0 , Stanojevic $M$, I vankoviæ $D$, Kerner $M$, Tikvica $A$. The comparison of fetal behavior in high-risk and normal pregnancies assessed by four-dimensional ultrasound. J M atern Fetal N eonatal M ed 2010 Dec;23(12):1461-67.

\section{ABOUT THE AUTHORS}

\section{Alin Başgül Yiğiter (Corresponding Author)}

Professor, Fetal M edicine, Consultant in the Perinatology, Chief of Perinatology, Department of M edical Faculty, Istanbul Bilim U niversity Istanbul, Turkey, e-mail: alinbasgul@gmail.com

\section{Gökçenur Gönenç}

Consultant, Department of Perinatology, M edical Faculty, Istanbul Bilim U niversity, Istanbul, Turkey

\section{Herman İşçi}

Assistant Professor, Department of Perinatology, Medical Faculty Istanbul Bilim University, Istanbul, T urkey

\section{Nilgün Güdücü}

Assistant Professor, Department of Perinatology, Medical Faculty Istanbul Bilim University, Istanbul, T urkey 